REPRESENTATION THEORY

An Electronic Journal of the American Mathematical Society

Volume 15, Pages 729-732 (December 16, 2011)

S 1088-4165(2011)00402-7

\title{
CORRIGENDUM TO “THE WEIL-STEINBERG CHARACTER OF FINITE CLASSICAL GROUPS"
}

\author{
G. HISS AND A. ZALESSKI
}

AbSTRACT. This paper corrects the statement and the proof of Theorem 1.5 of the paper quoted in the title (Represent. Theory 13 (2009), 427-459).

Theorem 1.5] of our paper [1] requires a correction. Below we provide a new statement of this theorem and correct the proof. A mistake in the original proof of Theorem 1.5 is due to missing the multiple 2 at a certain point of the proof (see [1, page 456, line 23]).

Let $\mathbf{G}$ be a simple algebraic group of type $C_{n}$ defined over a field of characteristic 2 and $G=S p(2 n, q), q=2^{k}$. If $\mu$ is a dominant weight of $\mathbf{G}$ then $\varphi_{\mu}$ denotes the irreducible representation of $\mathbf{G}$ with highest weight $\mu$, and $\Phi_{\mu}$ is the representation of $G$ afforded by the principal indecomposable module corresponding to $\left(\varphi_{\mu}\right)_{G}$ if $\mu$ is a $q$-restricted weight. In addition, $\omega:=\left(\varphi_{(q-1) \lambda_{n}}\right)_{G}$. Let st be the 2modular Steinberg representation of $G$. Recall that $s t=\left(\varphi_{(q-1)\left(\lambda_{1}+\cdots+\lambda_{n}\right)}\right)_{G}=$ $\Phi_{s t}$, where $\lambda_{1}, \ldots, \lambda_{n}$ are the fundamental weights of $\mathbf{G}$. The standard Frobenius endomorphism $\mathbf{G} \rightarrow \mathbf{G}$ is denoted by $F r_{0}$, and it acts on the representations and the weights of $\mathbf{G}$ (so $F r_{0}(\mu)=2 \mu$ ).

Theorem 1.5] in [1] has to be corrected as follows:

Theorem 1.5. Let $\lambda_{1}, \ldots, \lambda_{n}$ be the fundamental weights of $\mathbf{G}$, and let $\tau=$ $(q-1)\left(\lambda_{1}+\cdots+\lambda_{n-1}\right)$. Then $\omega \otimes s t=s t \oplus \Phi_{\tau}$.

Recall that $\varepsilon_{1}, \ldots, \varepsilon_{n}$ denote the weights of $\mathbf{G}$ introduced in [2, Planchee III]. The following lemma is a refinement of [1, Lemma 7.2(1)].

Lemma. $\varphi_{2 \lambda_{n}}$ is the only composition factor of $\varphi_{\lambda_{n}} \otimes \varphi_{\lambda_{n}}$ occurring with multiplicity 1 .

Proof. Let $M$ be the G-module afforded by the representation $\varphi_{\lambda_{n}} \otimes \varphi_{\lambda_{n}}$. Note that the weights of $\varphi_{\lambda_{n}}$ and hence of $M$ are known. In terms of $\varepsilon_{j}$ the weights of $\varphi_{\lambda_{n}}$ are $\pm \varepsilon_{1} \pm \cdots \pm \varepsilon_{n}$, so the weights of $M$ are $\sum_{i \in N} \pm 2 \varepsilon_{i}$, where $N$ can be any subset of $\{1, \ldots, n\}$ (possibly empty; in this case the weight in question is meant to be the zero weight). It follows that $2 \lambda_{i}=2 \varepsilon_{1}+\cdots+2 \varepsilon_{i}(i=1, \ldots, n)$ occur as weights of $M$.

Let $\mathbf{H}=G L\left(2 n, \bar{F}_{2}\right)$ and let $\varepsilon_{1}^{\prime}, \ldots, \varepsilon_{2 n}^{\prime}$ be the weights of the natural $\mathbf{H}$-module $V$. One can embed $\mathbf{G}$ into $\mathbf{H}$ so that a maximal torus $\mathbf{T}$ of $\mathbf{G}$ is contained in a maximal torus $\mathbf{T}^{\prime}$ of $\mathbf{H}$, and $\varepsilon_{i}=\left.\varepsilon_{i}^{\prime}\right|_{\mathbf{T}},\left.\varepsilon_{n+i}^{\prime}\right|_{\mathbf{T}}=-\varepsilon_{i}$ for $i=1, \ldots, n$. Let $V_{i}$ $(1 \leq i \leq 2 n)$ be the $i$-th exterior power of $V$, and $V_{0}$ the trivial $\mathbf{H}$-module. Set

Received by the editors October 17, 2010.

2000 Mathematics Subject Classification. Primary 20G40, 20C33.

Key words and phrases. Finite classical groups, Steinberg representation, Weil representation.

(C)2011 American Mathematical Society Reverts to public domain 28 years from publication 
$R=\bigoplus_{i=0}^{2 n} V_{i}$. Then the weights of $R$ are 0 and $\varepsilon_{j_{1}}^{\prime}+\cdots+\varepsilon_{j_{i}}^{\prime}$, where $1 \leq i \leq 2 n$ and $0<j_{1}<j_{2}<\cdots<j_{i} \leq 2 n$. It follows that the weights of $R^{\prime}:=R_{\mathbf{G}}$ are 0 and $\pm \varepsilon_{j_{1}} \pm \cdots \pm \varepsilon_{j_{i}}$ where $1 \leq i \leq n$ and $0<j_{1}<j_{2}<\cdots<j_{i} \leq n$. Therefore, the weights of $F r_{0}\left(R^{\prime}\right)$ and $M$ are the same.

Furthermore, 0 and $\lambda_{1}, \ldots, \lambda_{n}$ are the only dominant weights of $R^{\prime}$. Indeed, $\varepsilon_{1}=\lambda_{1}$ and $\varepsilon_{i}=\lambda_{i}-\lambda_{i-1}$ for $i>1$. Suppose that a non-zero weight $\sum_{i \in N} \pm \varepsilon_{i}=$ $\sum_{i \in N} \pm\left(\lambda_{i}-\lambda_{i-1}\right)$ is dominant. Then the coefficient of $\lambda_{i}$ is non-negative for every $i$. It follows that $1 \in N$ and if $i \in N, i>1$ then $i-1 \in N$. So $\sum_{i \in N} \pm \varepsilon_{i}$ is dominant if and only if it is of shape $\sum_{i=1}^{k} \varepsilon_{i}, k=1, \ldots, n$, as claimed. Therefore, if $\mu$ is the highest weight of an irreducible constituent $\varphi_{\mu}$ of $R^{\prime}$, then $\mu=\lambda_{i}$ for some $i$ or 0 .

Let $m_{i}^{\prime}(i=1, \ldots, n)$ be the multiplicity of $\varphi_{2 \lambda_{i}}$ in $F r_{0}\left(R^{\prime}\right)$ and $m_{i}$ the multiplicity of $\varphi_{2 \lambda_{i}}$ in $M$; in addition, let $m_{0}^{\prime}, m_{0}$ be the multiplicity of the trivial $G$-module in composition series of $F r_{0}\left(R^{\prime}\right), M$, respectively. We show that $m_{i}=m_{i}^{\prime}$. As the restriction $\varphi_{2 \lambda_{i}}$ to $S p(2 n, 2)$ is irreducible, and $\left(\varphi_{2 \lambda_{i}}\right)_{S p(2 n, 2)}$ and $\left(\varphi_{2 \lambda_{j}}\right)_{S p(2 n, 2)}$ are non-equivalent for $i \neq j$, it follows that $m_{i}^{\prime}$, respectively, $m_{i}$ is the multiplicity of the irreducible representation $\left(\varphi_{2 \lambda_{i}}\right)_{S p(2 n, 2)}$ in $S p(2 n, 2)$-composition series of $F r_{0}\left(R^{\prime}\right)$, respectively, $M$. Similarly, $m_{0}^{\prime}$ and $m_{0}$ is the multiplicity of the trivial $\operatorname{Sp}(2 n, 2)$ module in $F_{0}\left(R^{\prime}\right), M$, respectively. By [1, Lemma 7.2], the composition factors of $\bigoplus_{i=0}^{2 n}\left(V_{i}\right)_{S p(2 n, 2)}$ coincide with the composition factors of $\left(\varphi_{\lambda_{n}} \otimes \varphi_{\lambda_{n}}\right)_{S p(2 n, 2)}$ with regarding their multiplicities. It follows that $m_{0}^{\prime}=m_{0}$ and $m_{i}^{\prime}=m_{i}$ for $i=1, \ldots, n$.

Note that $\left(V_{i}\right)_{S p\left(2 n, \bar{F}_{2}\right)} \cong\left(V_{2 n-i}\right)_{S p\left(2 n, \bar{F}_{2}\right)}$ and $\left(V_{i}\right)_{S p\left(2 n, \bar{F}_{2}\right)}$ contains $\varphi_{\lambda_{i}}$ for $i=1, \ldots, n$. In addition, $\left(V_{0}\right)_{S p\left(2 n, \bar{F}_{2}\right)} \cong\left(V_{2 n}\right)_{S p\left(2 n, \bar{F}_{2}\right)}$. It follows that $\varphi_{\lambda_{n}}$ is the only composition factor of $R^{\prime}$ which may occur with multiplicity 1 . By general theory, $\varphi_{2 \lambda_{n}}$ does occur in $M$ with multiplicity 1.

Proof of Theorem 1.5. Let $\nu=a_{1} \lambda_{1}+\cdots+a_{n} \lambda_{n}$, where $0 \leq a_{1}, \ldots, a_{n} \leq q-1$, and $\nu^{\prime}=a_{1} \lambda_{1}+\cdots+a_{n-1} \lambda_{n-1}$. We show that $\Phi_{\nu}$ is a direct summand of $\omega \cdot s t$ if and only if $\nu=(q-1)\left(\lambda_{1}+\cdots+\lambda_{n}\right)$ or $\tau$. By [1, Lemma 7.4], it suffices to show that $s t$ is an irreducible constituent of $\left(\varphi_{\nu} \otimes \varphi_{(q-1) \lambda_{n}}\right)_{G}$ if and only if $\nu^{\prime}=\tau$ and $a_{n}=0$ or $q-1$.

It can be deduced from Steinberg [3, Corollary to Theorem 41 and Theorem 43] that $\varphi_{\nu}=\varphi_{\nu^{\prime}} \otimes \varphi_{a_{n} \lambda_{n}}$. In particular, $\varphi_{\nu^{\prime}} \otimes \varphi_{(q-1) \lambda_{n}}=\varphi_{\nu^{\prime}+(q-1) \lambda_{n}}$.

If $a_{n}=0$, then $\nu=\nu^{\prime}$ so the representation $\varphi_{\nu} \otimes \varphi_{(q-1) \lambda_{n}}=\varphi_{\nu^{\prime}+(q-1) \lambda_{n}}$ is irreducible. As $\nu^{\prime}+(q-1) \lambda_{n}$ is a $q$-restricted dominant weight, $\left(\varphi_{\nu^{\prime}+(q-1) \lambda_{n}}\right)_{G}$ is irreducible, so it is not equal to st unless $\nu^{\prime}=\tau$. If $\nu=\nu^{\prime}=\tau$, then $\left(\varphi_{\nu^{\prime}+(q-1) \lambda_{n}}\right)_{G}=s t$, so st is a direct summand of $\left(\varphi_{\nu} \otimes \varphi_{(q-1) \lambda_{n}}\right)_{G}\left(\right.$ when $\left.a_{n}=0\right)$.

Suppose that $a_{n}>0$. It follows from [1, Corollary 1.3] that every principal indecomposable module $\Phi_{\nu}$ occurs as a direct summand of $\left(\varphi_{(q-1) \lambda_{n}}\right)_{G} \otimes s t$ with multiplicity at most 1; by [1, Lemma 7.4], this implies that st occurs as an irreducible constituent of $\left(\varphi_{\nu} \otimes \varphi_{(q-1) \lambda_{n}}\right)_{G}$ with multiplicity at most 1 . Therefore, the constituents occurring with multiplicity greater than 1 can be ignored.

We have

$$
\varphi_{\nu} \otimes \varphi_{\lambda_{(q-1) \lambda_{n}}}=\varphi_{\nu^{\prime}} \otimes \varphi_{a_{n} \lambda_{n}} \otimes \varphi_{(q-1) \lambda_{n}} .
$$

We show that a composition factor of $\left(\varphi_{a_{n} \lambda_{n}} \otimes \varphi_{(q-1) \lambda_{n}}\right)_{G}$ has multiplicity greater than 1 , unless $a_{n}=q-1$, and it is of form $\left(\varphi_{(q-1) \lambda_{n}}\right)_{G}$. 
Let $a_{n}=\sum_{i=0}^{k-1} 2^{i} b_{i}$ be the 2 -adic expansion of $a_{n}$ (so $\left.0 \leq b_{i} \leq 1\right)$. Then

$\varphi_{a_{n} \lambda_{n}} \otimes \varphi_{(q-1) \lambda_{n}}=\left(\varphi_{b_{0} \lambda_{n}} \otimes \varphi_{\lambda_{n}}\right) \otimes F r_{0}\left(\varphi_{b_{1} \lambda_{n}} \otimes \varphi_{\lambda_{n}}\right) \otimes \cdots \otimes F r_{0}^{k-1}\left(\varphi_{b_{k-1} \lambda_{n}} \otimes \varphi_{\lambda_{n}}\right)$.

If $b_{i}=0$, then $\varphi_{b_{i} \lambda_{n}} \otimes \varphi_{\lambda_{n}}=\varphi_{\lambda_{n}}$, otherwise $b_{i}=1$ and then, by the lemma above, the only composition factor of $\left(\varphi_{b_{i} \lambda_{n}} \otimes \varphi_{\lambda_{n}}\right)_{G}$ occurring with multiplicity 1 is $\left(\varphi_{2 \lambda_{j}}\right)_{G}$ for $1 \leq j \leq n$. Therefore, the composition factors of $\varphi_{\lambda_{n}} \otimes \varphi_{\lambda_{n}}$ distinct from $\varphi_{2 \lambda_{n}}$ can be ignored. This means that we have to decide whether st occurs as a composition factor of the representation obtained from

$$
\left(\varphi_{\nu^{\prime}} \otimes \varphi_{b_{0} \lambda_{n}} \otimes \varphi_{\lambda_{n}} \otimes \cdots \otimes F r_{0}^{k-1}\left(\varphi_{b_{k-1} \lambda_{n}} \otimes \varphi_{\lambda_{n}}\right)\right)_{G}
$$

by omitting $\varphi_{b_{i} \lambda_{n}}$ whenever $b_{i}=0$, and replacing $\varphi_{b_{i} \lambda_{n}} \otimes \varphi_{\lambda_{n}}$ by $\varphi_{2 \lambda_{n}}$ whenever $b_{i}=1$. Let $B=\left\{i \in\{0, \ldots, k-1\}: b_{i}=1\right\}$.

Then we can write the resulting expression as

$$
\left(\otimes_{i \in B} F r_{0}^{i}\left(\varphi_{2 \lambda_{n}}\right) \otimes_{i \notin B} F r_{0}^{i}\left(\varphi_{\lambda_{n}}\right)\right)_{G}=\left(\otimes_{i \in B} F r_{0}^{i+1}\left(\varphi_{\lambda_{n}}\right) \otimes_{i \notin B} F r_{0}^{i}\left(\varphi_{\lambda_{n}}\right)\right)_{G} .
$$

We first consider the set $B^{\prime}:=\{i \in B: i+1 \notin B\}$ (if $i=k-1$, then $i+1$ is regarded to be 0 ). Suppose that $B^{\prime}$ is non-empty (this means that $a_{n} \neq 0$ and $a_{n} \neq 2^{k}-1$ ). For $i \in B^{\prime}$ the lemma above applied to the term $\left(F r_{0}^{i+1}\left(\varphi_{\lambda_{n}}\right) \otimes \operatorname{Fr}_{0}^{i+1}\left(\varphi_{\lambda_{n}}\right)\right)_{G}=$ $\left(F r_{0}^{i+1}\left(\varphi_{\lambda_{n}} \otimes \varphi_{\lambda_{n}}\right)\right)_{G}$ tells us that, by the above reason, we can replace $\varphi_{\lambda_{n}} \otimes \varphi_{\lambda_{n}}$ by $\varphi_{2 \lambda_{n}}$ so $\left(F r_{0}^{i+1}\left(\varphi_{\lambda_{n}} \otimes \varphi_{\lambda_{n}}\right)\right)_{G}$ is replaced by $\left(F r_{0}^{i+2}\left(\varphi_{\lambda_{n}}\right)\right)_{G}\left(i \in B^{\prime}\right)$. One can continue the analysis by repeating this reasoning, but it is more efficient to observe that the process is parallel to the addition of the residues of integers modulo $2^{k}-1$. In order to justify this claim, we first compute $\left(\varphi_{a_{n} \lambda_{n}} \otimes F r_{0}^{i}\left(\varphi_{\lambda_{n}}\right)_{G}\right.$ for every $i=0, \ldots, k-1)$, and then in general.

Note that

$$
\left(\varphi_{a_{n} \lambda_{n}} \otimes \varphi_{2^{i} \lambda_{n}}\right)_{G}=\left(F_{0}^{i}\left(\varphi_{b_{i} \lambda_{n}} \otimes \varphi_{\lambda_{n}}\right) \otimes_{j \neq i} \varphi_{2^{j} b_{j} \lambda_{n}}\right)_{G}
$$

If $b_{i}=0$, then $\left(\varphi_{b_{i} \lambda_{n}}\right)_{G}=1_{G}$ so $F r_{0}^{i}\left(\varphi_{b_{i} \lambda_{n}} \otimes \varphi_{\lambda_{n}}\right)_{G}=\left(F r_{0}^{i}\left(\varphi_{\lambda_{n}}\right)\right)_{G}$. Suppose that $b_{i}=1$. In view of the lemma above, the composition factors of $\varphi_{b_{i} \lambda_{n}} \otimes$ $\varphi_{\lambda_{n}}$ other than $\varphi_{2 \lambda_{n}}$ occur with multiplicity greater than 1 , so they are immaterial for our purpose. Therefore, we are left with $\varphi_{2 \lambda_{n}}$, so it suffices to compute $\left(F_{0}^{i}\left(\varphi_{2 \lambda_{n}}\right) \otimes_{j \neq i} \varphi_{2^{j} b_{j} \lambda_{n}}\right)_{G}$. This is equal to $\left(F r_{0}^{i+1}\left(\varphi_{\lambda_{n}}\right) \otimes_{j \neq i} \varphi_{2^{j} b_{j} \lambda_{n}}\right)_{G}$. If $i+1=k$, then $\left(F r_{0}^{k}\left(\varphi_{\lambda_{n}}\right)\right)_{G}=\left(\varphi_{2^{k} \lambda_{n}}\right)_{G} \cong\left(\varphi_{\lambda_{n}}\right)_{G}$, and the replacement of $\left(\varphi_{2^{k} \lambda_{n}}\right)_{G}$ by $\left(\varphi_{\lambda_{n}}\right)_{G}$ is parallel to taking the residue modulo $2^{k}-1$. Next, if $b_{i+1}=0$, then we stop, otherwise we repeat the same trick and obtain $\left(F r_{0}^{i+2}\left(\varphi_{\lambda_{n}}\right) \otimes_{j \neq i, i+1} \varphi_{2^{j} b_{j} \lambda_{n}}\right)_{G}$. The output of the procedure will be $\varphi_{a_{n}(i)}$, where $a_{n}(i)=\left(a_{n}+2^{i}\right)\left(\bmod 2^{k}-1\right)$.

In general, applying this to $\varphi_{a_{n} \lambda_{n}} \otimes \varphi_{(q-1) \lambda_{n}}=\left(\varphi_{a_{n} \lambda_{n}} \otimes_{i=0}^{k-1} \operatorname{Fr}_{0}^{i}\left(\varphi_{\lambda_{n}}\right)\right)_{G}$, we obtain $\left(\varphi_{a_{n}^{\prime} \lambda_{n}}\right)_{G}$, where $a_{n}^{\prime}=a_{n}+1+2+\cdots+2^{k-1}\left(\bmod 2^{k}-1\right)=a_{n}$.

This is also true if $B^{\prime}$ is empty but $a_{n}=q-1$. Indeed, the reasoning above for $i=0, \ldots, k-1$ remains valid, and we obtain $a_{n}(i)=\left(a_{n}+2^{i}\right)\left(\bmod 2^{k}-1\right)=2^{i}$. Then again $a_{n}^{\prime}=a_{n}=q-1$.

Thus, we conclude that it suffices to decide whether st is an irreducible constituent of $\left(\varphi_{\nu^{\prime}} \otimes \varphi_{a_{n} \lambda_{n}}\right)_{G}$. As mentioned above, $\varphi_{\nu^{\prime}} \otimes \varphi_{a_{n} \lambda_{n}}=\varphi_{\nu^{\prime}+a_{n} \lambda_{n}}$. Since $\nu^{\prime}+a_{n} \lambda_{n}$ is a $q$-restricted dominant weight, $\left(\varphi_{\nu^{\prime}+a_{n} \lambda_{n}}\right)_{G}$ is irreducible. So st is an irreducible constituent of $\left(\varphi_{\nu^{\prime}+a_{n} \lambda_{n}}\right)_{G}$ if and only if $\left(\varphi_{\nu^{\prime}}+a_{n} \lambda_{n}\right)_{G}=s t$, equivalently, $\nu^{\prime}+a_{n} \lambda_{n}=(q-1)\left(\lambda_{1}+\cdots+\lambda_{n}\right)$. This implies $\nu^{\prime}=(q-1)\left(\lambda_{1}+\cdots+\lambda_{n-1}\right)=\tau$ and $a_{n}=q-1$.

Therefore, $\omega \otimes s t=\Phi_{\tau} \oplus s t$, as required. 
The comments to Theorem 1.5 in [1, page 430, line -12] concerning the decomposition numbers of $\omega \cdot S t$ cannot be applied to the new version of the theorem. In fact, we have:

Corollary. $\operatorname{dim} \Phi_{\tau}=|G|_{2} \cdot\left(\operatorname{dim} \varphi_{(q-1) \lambda_{n}}-1\right)=|G|_{2} \cdot\left(q^{n}-1\right)$, and the ordinary character corresponding to $\Phi_{\tau}$ is multiplicity free.

Proof. $\left(\varphi_{(q-1) \lambda_{n}}\right)_{G} \otimes s t=\Phi_{\tau} \oplus s t$, so $\operatorname{dim} \Phi_{\tau}=\operatorname{dim} s t \cdot\left(\operatorname{dim} \varphi_{(q-1) \lambda_{n}}-1\right)$. As $\operatorname{dim} s t=|G|_{2}$ and $\operatorname{dim} \varphi_{(q-1) \lambda_{n}}=2^{n k}=q^{n}$, the first claim follows. The second one follows from [1, Corollary 1.3].

Example. Let $G=S p(2, q), q$ even. Then $\tau=0$; by the above corollary, $\operatorname{dim} \Phi_{0}=$ $q(q-1)$.

Example. Let $G=S p(4,4)$, so $n=2$. Then $\tau=3 \lambda_{1}$ and $|G|_{2}=2^{8}=256$. So $\operatorname{dim} \varphi_{3 \lambda_{2}}=4^{2}=16$. By the corollary above, $\operatorname{dim} \Phi_{\tau}=256 \cdot 15=3840$.

\section{REFERENCES}

[1] G. Hiss and A.E. Zalesski, The Weil-Steinberg character of finite classical groups with an appendix by Olivier Brunat, Represent. Theory 13 (2009), 427-459. MR2550472 (2010m:20016)

[2] N. Bourbaki, Groupes et algebres de Lie, Chaps. IV-VI, Hermann, Paris, 1968. MR0240238 $(39: 1590)$

[3] R. Steinberg, Lectures on Chevalley groups, mimeographed lecture notes, Yale Univ. Math. Dept., New Haven, CT., 1968. MR0466335 (57:6215)

Lehrstuhl D für Mathematik, RWTH Aachen University, 52056 Aachen, Germany

E-mail address: gerhard.hiss@math.rwth-achen.de

Dipartimento di Matematica e Applicazioni, Università degli Studi di Milano-Bicocca, via R. Cozzi 53, 20126 Milano, Italy

E-mail address: alexandre.zalesski@gmail.com 